\title{
Importance of aberration theory in understanding lens design
}

\section{Michael Kidger}

Michael J. Kidger, "Importance of aberration theory in understanding lens design," Proc. SPIE 3190, Fifth International Topical Meeting on Education and Training in Optics, (8 December 1997); doi: 10.1117/12.294396

Event: Fifth International Topical Meeting on Education and Training in Optics, 1997, Delft, Netherlands 


\title{
The Importance of Aberration Theory in Understanding Lens Design
}

\author{
Michael J Kidger \\ Kidger Optics Ltd., \\ 9a, High Street, Crowborough, \\ East Sussex, TN6 2QA, United Kingdom
}

\begin{abstract}
Introduction
The successful design of optical imaging systems requires many skills. In this paper we are considering 'Lens Design', the design of the refracting (or reflecting, or sometimes diffracting) surfaces in an optical imaging system, to give the best possible image quality.
\end{abstract}

To be effective in lens design, it is necessary to understand geometrical optics, aberration theory, physical optics and image formation, and to be able to use optical design software and to know what optical systems have been used successfully in the past. In addition some understanding of optical testing and the properties of sources and detectors is also necessary.

After teaching lens design for over 30 years, I have come to the conclusion that, while other topics are clearly important, the central subject, that must be well understood, is aberration theory. Without a good understanding of aberration theory the designer will simply try one 'trial-and-error' solution after another, possibly even using a computer program to generate these solutions automatically

In our courses, we teach students about Seidel aberrations, and about other topics which help understanding. We concentrate on explaining which aberrations are introduced by individual surfaces, or by individual groups of surfaces; from this the designer should appreciate which types of design have the potential to give good performance, and can use lens design software intelligently.

\section{Background}

In my career in Optics, I have been fortunate in having some incredible teachers.

My initial interest started from an early interest in photography, and as an undergraduate student at Imperial College I was very lucky to be tutored by Professor W.T.Welford (Reference 1). Later he taught me geometrical optics and thin film optics, and much later, when I was teaching optical design at Imperial College, I was privileged to have many fruitful discussions with him.

As a postgraduate student at Imperial College, I was lucky to be taught image formation, aberration theory and lens design by Professor H.H.Hopkins (Reference 2). He had developed the techniques of A.E.Conrady (Reference 3), who had taught lens design at Imperial College much earlier. His work on early zoom lenses for television, and on rod-lens endoscopes was outstanding.

Later, I joined the Optical Design Group at Imperial College under the supervision of Professor C.G.Wynne. His expertise in lens design is well-known, and he was one of the pioneers of optimisation in lens design. He has an outstanding insight into lens design and aberrations.

Only a small number of people have had the privilege of being taught by these three outstanding people, and in my teaching I have tried to take the best features from each of them. 
After a few years in the Optical Design Group, I took over the task of teaching lens design at Imperial College. Later, when we formed Kidger Optics, I continued to teach lens design, both in our own courses, and in short courses, as part of SPIE symposia. I have given courses in lens design in Australia, England, Finland, France, Germany, Italy, Korea, Netherlands, Singapore, Sweden and USA.

Although optical designers (like the rest of us) now have to be computer-literate, the most important requirement is some knowledge of optics, and particularly of aberration theory and its applications. It is much easier to teach lens design to a person who know about optics, even if they don't know how to switch a computer on, than to teach lens design to a computer expert who knows no optics.

\section{WHAT DO LENS DESIGNERS NEED TO KNOW ABOUT?}

In reality people learn best by doing the job. But to give someone a lens design program and expect them to sort it out for themselves is both unreasonable and inefficient. If they are very good they will re-discover things that were already well-known, and if they are not very good, they will find it very difficult.

It is over 100 years since H.D. Taylor designed the Cooke triplet, and P.Rudolph designed the Planar, and there is a lot that is known, and can be taught, that will provide a framework to which an intelligent person can add their later experiences. So I see it as my job, when teaching lens design, to provide this framework. But what, precisely, is needed?

\section{Paraxial Optics}

When I started teaching lens design, at Imperial College, I was teaching as part of the M.Sc. degree course. In a university course, at the end of the year, the students will take an examination. So the course is expected to be mathematically rigorous, with plenty of mathematics. But in real lens design you rarely have to derive results in paraxial optics, and now I include only an extremely small amount of paraxial optics in my courses. Topics that need to be understood are:-

a The paraxial approximation

b Paraxial ray tracing

c The Lagrange invariant

d Paraxial properties such as focal length, magnification, pupil positions

\section{Aberrations}

This is the central topic, that interests me, and which is so essential, and which is the main topic of this paper. We start with a discussion of the properties of the Seidel aberrations. We also have to show how each of these aberrations appear when plotted in the various ways that we see in the literature, and by optical design software. So this includes some discussion of:-

$\begin{array}{ll}\text { a } & \text { Aberration curves } \\ \text { b } & \text { Spot diagrams } \\ \text { c } & \text { Wavefront aberration plots } \\ \text { d } & \text { Effects on image quality }\end{array}$

We have to explain that transverse aberrations are proportional to the slope of a wavefront aberration plot, and depending on the time available and on the interests of the students, we can spend one hour, or many hours, discussing all of these things. We also have to explain that if we think primarily in terms of wavefront aberrations, one major advantage is that the aberrations of a complete lens are simply the sums of the aberrations of the surfaces. This makes it possible to easily identify which surfaces in a lens affect the aberrations, and how they do this. 


\section{ABERRATIONS OF SINGLE SURFACES}

This is the key to understanding lens design, and the only way that I can explain what we do is to include a short extract from our course notes:-

Theory of Seidel Aberrations - Surface Contributions

In order to calculate the Seidel aberrations, we first trace a paraxial marginal ray and a paraxial chief ray.

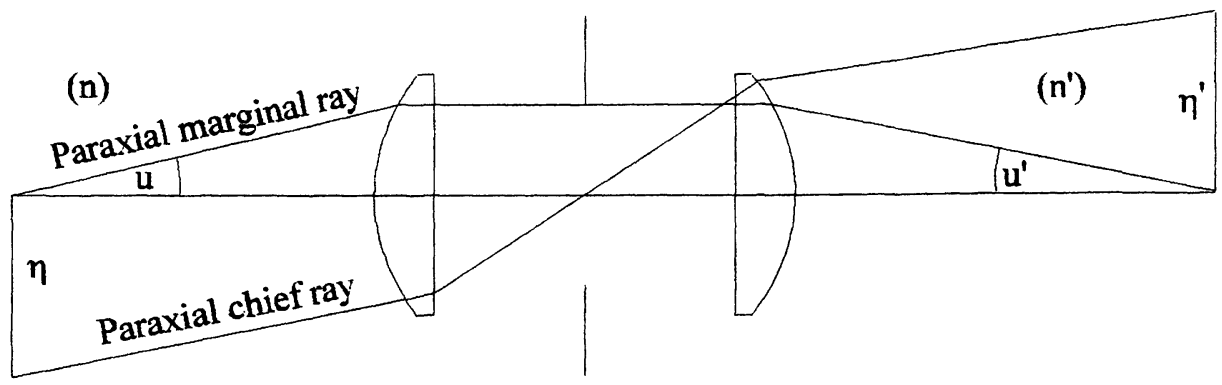

\section{Paraxial Marginal Ray and Chief Ray}

For these two rays, we calculate, at each surface, the following quantities:-

$\mathrm{A}$ is $\mathrm{ni}$, where $\mathrm{i}$ is the angle of incidence of the paraxial marginal ray

$h$ is the paraxial marginal ray height

$\bar{A}$ is $n \bar{i}$, where $\bar{i}$ is the angle of incidence of the paraxial chief ray.

$\mathrm{H}$ is the Lagrange invariant, $\mathrm{n} u \mathrm{\eta}$.

$\partial \mathrm{n}$ is the dispersion of the material, conventionally $n_{F}-n_{C}$, but any other wavelengths can be used.

The contributions of each surface to the Seidel aberrations are:-

\begin{tabular}{|c|c|c|c|}
\hline Spherical aberration & $S_{1}$ & $=$ & $-A^{2} h\left(u^{\prime} / n^{\prime}-u / n\right.$ \\
\hline Coma & $S_{2}$ & $=$ & $-\mathbf{A} \bar{A} h\left(u^{\prime} / n^{\prime}-u /\right.$ \\
\hline Astigmatism & $\mathbf{S}_{3}$ & $=$ & $-\bar{A}^{2} 1$ \\
\hline Field curvature & $\mathrm{S}_{4}$ & $=$ & $-\mathrm{H}^{2} \mathrm{c}$ \\
\hline Distortion & $\mathbf{S}_{5}$ & $=$ & $(\overline{\mathrm{A}} / \mathbf{A})\left(\mathrm{S}_{3}+\mathrm{S}_{4}\right)$ \\
\hline Axial colour & $\mathrm{C}_{1}$ & $=$ & $\operatorname{Ah}\left(\partial n^{\prime} / n^{\prime}-\partial n / n\right)$ \\
\hline ateral colour & $\mathrm{C}_{2}$ & $=$ & $\bar{A} h / \partial n^{\prime}$ \\
\hline
\end{tabular}

The total aberration of the lens is the sum of the surface contributions. 


\section{Conditions for Zero Aberration at a Surface}

It is possible to construct a design in which some of the Seidel aberrations are zero at some surfaces, and although this is very rarely a useful way of designing real lenses, it shows how specific aberrations can be corrected.

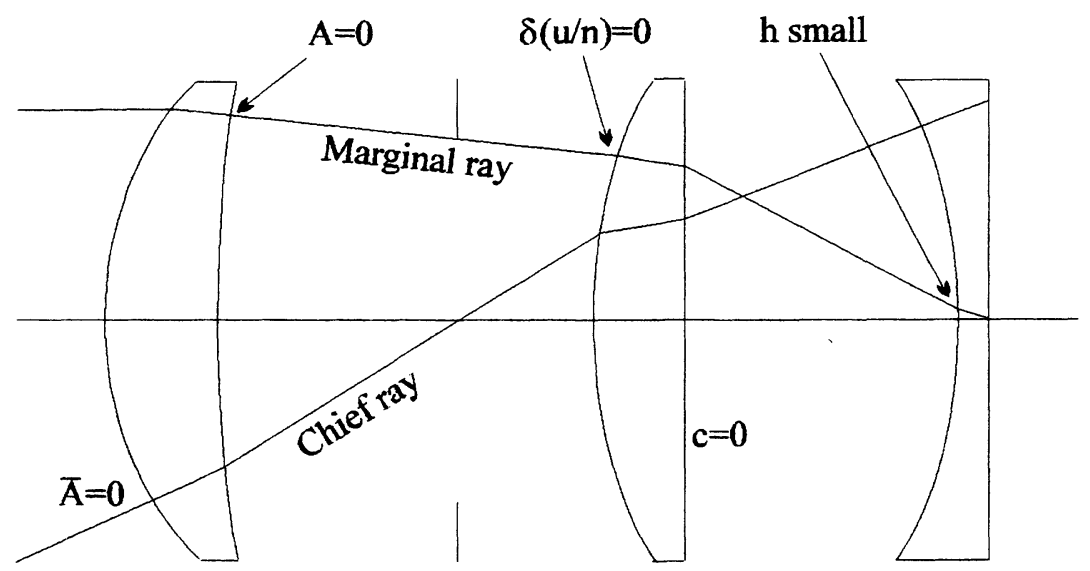

Conditions for Zero Aberration

In the above diagram, the position of the entrance pupil has been chosen so that the chief ray angle of incidence $(\bar{A})$ at surface 1 is zero, making $S_{2}, S_{3}, S_{5}$ and $C_{2}$ all zero at that surface.

At surface 2, the marginal ray angle of incidence (A) is zero, making $S_{1}, S_{2}$ and $C_{1}$ all zero at that surface.

Ignoring the stop surface, surface 3 satisfies the 'aplanatic condition' which is that $u^{\prime} / n^{\prime}=u / n$. When this condition is satisfied, $S_{1}, S_{2}$ and $S_{3}$ are all zero at that surface.

At surface 4, the curvature of the surface is zero. Perhaps surprisingly, the only aberration that is zero at this surface is the field curvature term, $S_{4}$. ALL other Seidel aberrations are non-zero.

Surface 5 is close to the image surface, so that all of the aberrations that depend on the marginal ray height, $\mathrm{h}$, are small. (They would be exactly zero if $h=0$, but this condition is rarely satisfied, because if an image is coincident with a surface, any surface defects will be in focus, and this is almost always unacceptable.) However, the field curvature term, $S_{4}$, is negative, which allows us to correct the positive field curvature of the rest of the system. At surface 6, if the plane surface is in contact with the image surface, all of the aberrations are zero. All of this is summarised in the table below:-

\begin{tabular}{|l|l|l|l|l|l|l|l|l|}
\hline & & $\mathrm{S}_{1}$ & $\mathrm{~S}_{2}$ & $\mathrm{~S}_{3}$ & $\mathrm{~S}_{4}$ & $\mathrm{~S}_{5}$ & $\mathrm{C}_{1}$ & $\mathrm{C}_{2}$ \\
\hline & & & & & & & & \\
\hline Surface 1 & $\overline{\mathrm{A}}=0$ & & 0 & 0 & & 0 & & 0 \\
\hline & & & & & & & & \\
\hline Surface 2 & $\mathrm{A}=0$ & 0 & 0 & & & & 0 & \\
\hline & & & & & & & & \\
\hline Surface 3 & $\mathbf{u}^{\prime} / \mathbf{n}^{\prime}-\mathrm{u} / \mathrm{n}=0$ & 0 & 0 & 0 & & & & \\
\hline & & & & & & & & \\
\hline Surface 4 & $\mathrm{c}=0$ & & & & 0 & & & \\
\hline & & & & & & & & \\
\hline Surface 5 & $\mathrm{h}$ small & small & small & small & large & small & small & small \\
\hline & & & & & & & & \\
\hline Surface 6 & $\mathrm{c}=0, \mathrm{~h}$ small & 0 & 0 & 0 & 0 & 0 & 0 & 0 \\
\hline
\end{tabular}


This illustrates that it is not possible for the seven Seidel aberrations to be corrected at all surfaces of a lens, and therefore aberration correction is almost always achieved by balancing positive aberrations against negative aberrations.

Of course there are other topics:-

i Aberrations of thin lenses

ii Petzval sum and how to correct it

iii The distinction between shape-dependent and shape-independent aberrations

iv Stop-shift effects

$v \quad$ The Abbe sine condition

vi Uses of symmetry (monocentric lenses and lenses with front-back symmetry)

vii Mirror surfaces

viii Aspheric surfaces

For a description of what these topics involve, see my paper "Principles of Lens Design" (Reference 4).

Depending on the time available, and on the interests of participants, we can then show how these basic principles are applied to the design of the following types of optical system:-

$\begin{array}{ll}1 & \text { Doublets } \\ 2 & \text { Eyepieces } \\ 3 & \text { Petzval lenses } \\ 4 & \text { Visual afocal systems } \\ 5 & \text { Thermal Imagers } \\ 6 & \text { Laser beam expanders } \\ 7 & \text { Photographic lenses } \\ 8 & \text { Microscope objectives } \\ 9 & \text { Other high aperture lenses } \\ 10 & \text { Wide-angle lenses } \\ 11 & \text { Apochromatic lenses } \\ 12 & \text { F.theta lenses } \\ 13 & \text { Apochromats } \\ 14 & \text { Catadioptric systems } \\ 15 & \text { Astronomical telescopes } \\ 16 & \text { Zoom lenses } \\ 17 & \text { Lenses for laser focussing and collimating. }\end{array}$

Although all of these topics introduce some special problems, in many ways they can all basically be regarded as examples of the application of simple aberration theory, and this is what we try to do. In this way, a lecture on one topic should contain material that is informative for all of the course participants. 


\section{SOME SIMPLE EXAMPLES WHERE SOME KNOWLEDGE HELPS}

\section{a Seidel Aberrations of Surfaces \\ 1 Petzval Sum}

One very obvious point that can be seen immediately from the Petzval sum, is that a convex air-glass surface introduces positive Petzval sum (i.e. a positive wavefront aberration) but a concave air-glass surface introduces negative Petzval sum So any lens which has a flat field must include at least one concave surface.

You can therefore see, immediately, that a simple eyepiece such as I have shown here, can not possibly have a flat field.

\section{Spherical Aberration $\left(\mathbf{S}_{\mathbf{1}}\right)$}

With a little more thought, we can understand the properties of each surface of a simple Fraunhofer doublet. Remember that the spherical aberration of a single surface is given by:-

$$
S_{1}=-A^{2} h\left(u^{\prime} / n^{\prime}-u / n\right)
$$

Looking at surface 1 we can see that $u=0$, but $u^{\prime}<0$. From this we can see that $u^{\prime} / n^{\prime}-u / n<0$ and therefore at surface $1, S_{1}>0$.

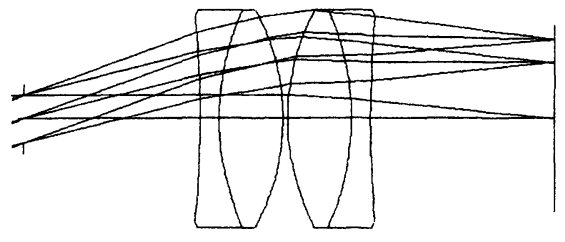

At surface 3, $\mathbf{u}<0$ and $\mathbf{u}^{\prime}<0$. But it is clear that at this surface, for a normal doublet, $u^{\prime} / \mathrm{n}^{\prime}-\mathrm{u} / \mathrm{n}<0$ and therefore at this surface $S_{1}>0$.

Therefore, to correct the total $S_{1}$ for the lens, the cemented surface 2 must have negative $S_{1}$ and to do this, $u^{\prime} / n^{\prime}-u / n>0$. Given that surface 2 has a negative curvature, we must choose glasses so that $n^{\prime}>n$. If we do this, automatically $u>u^{\prime}$, and therefore and therefore $S_{1}<0$.

This is not, of course, new, but it is included as an illustration of something that can be worked out, from first principles, without ANY calculation.

b Seidel Aberrations of Thin Lenses

One example that $\mathrm{I}$ like to use is a simple catadioptric system. It is typical of a class of "Mirror lenses" that are used in photography and for other applications, but it is more simple than most.

I try to explain the function of each component. I tabulate the aberrations of each component (on the next page), but for the purposes of understanding I visualise that the Mangin mirror consists of a meniscus lens, and a separate mirror surface. (Clearly, in reality, the mirror surface is actually the convex surface of the Mangin.)

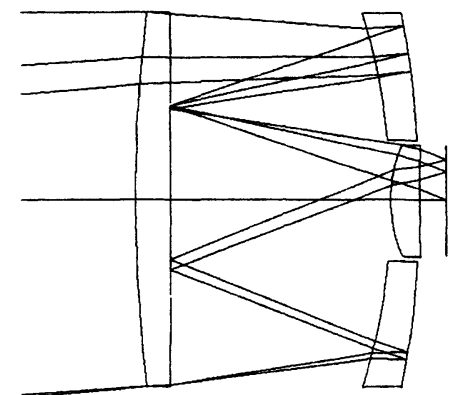


The following table shows the aberration contributions of each component, treating the Mangin mirror as a negative lens, followed by the mirror, followed by another negative lens.

$\begin{array}{lrcccccc} & S 1 & S 2 & S 3 & S 4 & S 5 & C 1 & C 2 \\ \text { Lens 1 } & .0257 & .0 & .0080 & .0050 & .0 & .0576 & .0001 \\ \text { Meniscus } & -.3610 & .0262 & -.0007 & -.0040 & -.0007 & -.0297 & -.0019 \\ \text { Mirror } & .5255 & -.0084 & .0244 & -.0427 & .0042 & .00 & .0 \\ \text { Meniscus } & -.2400 & .0353 & .0024 & -.004 & -.0007 & -.0273 & -.0020 \\ \text { Small lens } & .0570 & .0402 & -.0300 & .0443 & -.0191 & .0006 & .0057 \\ \text { Total } & .0071 & -.0067 & .0021 & -.0013 & -.0163 & .0013 & .0019\end{array}$

We see that the negative meniscus lens corrects the positive $S_{1}$ of the mirror. The front lens (Lens 1$)$ corrects the axial colour of the Mangin mirror. We can also see that the Mangin introduces $S_{2}$, which is corrected by the small lens. The $S_{3}$ and $S_{4}$ of the mirror are mainly corrected by the small lens. It is a very nice example, to illustrate the balancing of aberrations by the different components in the system.

\section{c The Sine Condition.}

This is a topic that I taught at university for 20 years. When I started teaching short courses I dropped it, but I later realised that there is a class of lens design problems that can be understood with reference to the sine condition, that are much more difficult to understand in any other way. I will give a very simple example:-

According to the Abbe sine condition, if spherical aberration is corrected, for a system working at a finite nonzero magnification, coma is corrected if (and only if)

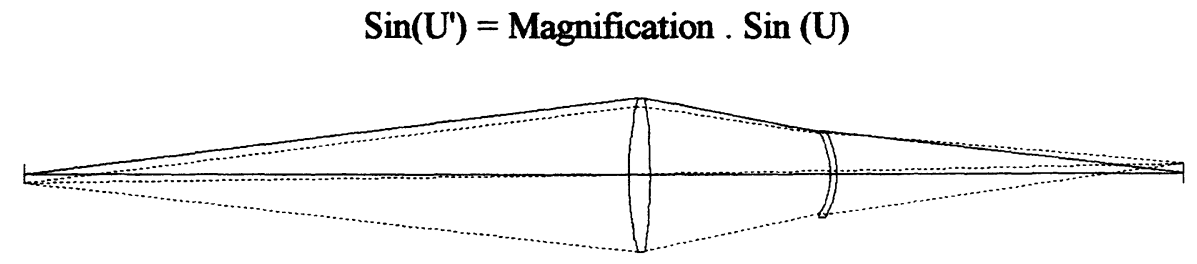

where $U$ is the exact ray angle of the marginal ray in the object space and $U^{\prime}$ is the exact angle in the image space.

But suppose we have a system that consists of two lenses with a large separation, as shown above, where the magnification is -1.0. It is possible to correct the spherical aberration, but the positive lens (in the system shown above) will have positive spherical aberration. Therefore the marginal ray will hit lens 2 at a height that is too small, and Sin(U') will be too small to satisfy the Sine Condition. So we can predict that, in a system as shown above, coma can not be corrected.

This also helps the designer to understand what can be done to correct the Sine Condition. There are several obvious possibilities:-

1 Reduce the separation between the lenses.

2 Make one lens aspheric, so that the spherical aberration is smaller. (This is not a complete solution.)

3 Make one, or both, lenses thicker. The lenses will probably have to be very thick, for this solution to help.

In this problem, the Sine Condition is the simplest way of understanding why the coma is not correctable. Without this understanding, a designer can spend many hours, trying to get a result, by optimisation, that is simply not possible. 
There are several other lens design problems where some understanding of the Sine Condition tells the designer when coma is correctable, and when it isn't.

\section{UNIVERSITY OR SHORT COURSES LATER?}

Having taught lens design in three situations, I think that I am qualified to express an opinion about the relative advantages and disadvantages.

\section{a Postgraduate Course at University}

As a graduate of the Imperial College M.Sc. course in Applied Optics, I have to say that the background knowledge that one can get from a one- or two-year course is tremendous. In my case I was primarily interested in lens design and image formation, but topics that did not much concern me at the time, such as colour vision and colour measurement, and thin-film optics, later became very useful. So I very strongly support courses like this.

But at the same time there are disadvantages. Most M.Sc. students come immediately after completing a B.Sc., and they usually have no experience in optics, so the subject is new to them, and they can't judge what will be most useful to them in the future. But sometimes there are students who come, after several years industrial experience, and the difference is amazing. When they are good, they understand the relevance of what they can learn in the course, and they gain much more from it.

\section{b One-week courses}

Most of my teaching is now done in one-week courses, run by Kidger Optics, and there are several advantages. Compared with an M.Sc. course, participants are motivated by the need to actually DO lens design, and they have usually spent some time trying to understand the subject before they come on the course. We can concentrate on teaching what we think is useful, rather than what can be tested in examinations.

The only disadvantage is really time - it is not always possible for prospective students to take a week away from work, and the cost is also important in some cases. On the other hand there is much more that is useful, and can be taught, than is possible in one week. Our solution is to provide courses at several levels, and many people have attended two (or more) of our courses

\section{c Shorter courses}

It is also possible to learn about lens design in short courses (from half a day, to two days) at SPIE conferences, mostly in the USA. The advantages of these are obvious. There are a wide variety of courses, and most people can manage one or two days away from work. But they are necessarily limited by the time available, and one or two days of lectures is tiring both for the instructor and the student. Where they are available, they provide a tremendous on-going opportunity for people to continue their education in all areas of optics and optical engineering.

\section{CONCLUSION}

Lens design is a 'mature' subject, but it is also of continuing importance. Optical design software is now very powerful, but a designer with some understanding of the physics involved in correcting aberrations, and of the effect of the aberrations on lens performance, is in a much better position to design lenses effectively.

\section{REFERENCES}

1 Aberrations of Optical Systems, W.T.Welford, Adam Hilger, 1986

2 The Wave Theory of Aberrations, H.H.Hopkins, Oxford University Press, 1950

$3 \quad$ Applied Optics and Optical Design, A.E.Conrady, Dover, 1957, 1960

4 Principles of Lens Design, M.J.Kidger, Critical Review 41, SPIE, 1992 